\title{
Initial peri- and postoperative antibiotic treatment of infected nonunions: results from 212 consecutive patients after mean follow-up of 34 months
}

This article was published in the following Dove Press journal:

Therapeutics and Clinical Risk Management

Lars Helbig'

Maren Bechberger ${ }^{2}$

Riyadh Aldeeri'

Adriana Ivanova'

Patrick Haubruck'

Matthias Miska'

Gerhard Schmidmaier'

Georg W Omlor'

'Department of Orthopaedics, Trauma Surgery and Paraplegiology, ${ }^{2}$ Pharmacy Department, Heidelberg University

Hospital, Heidelberg, Germany
Correspondence: Georg W Omlor Department of Orthopaedics, Trauma Surgery and Paraplegiology, Heidelberg University Hospital, Schlierbacher Landstrasse 200a, D-69118,

Heidelberg, Germany

Tel +49622I 5635378

Fax +496221969288

Email georg.omlor@med.uni-heidelberg.de
Purpose: Infected nonunions of the long bones belong to the most feared complications in the field of orthopedic and trauma surgery. Optimal antibiotic therapy should start early with the first revision surgery. Therefore, the aim of this study was to evaluate our peri- and postoperative antibiotic regime in context with the microbial spectrum and antibiotic resistances of patients with infected nonunions and to assess the possible impact on healing rates.

Methods: We included all patients with first revision surgery during 2010-2015 due to nonunion of long bones with a clinical history of infection treated with radical debridement, local application of a gentamicin-impregnated bone cement, and systemic cefuroxime. Mean follow-up was 34.2 months. Data collection was performed retrospectively using a computerized databank with information about microbial species from intraoperatively acquired tissue samples and respective antibiograms. Bone fusion rates were evaluated based on findings of the latest X-rays and computed tomography scans.

Results: Two hundred and twelve patients with nonunion and history of infection were selected; 171 patients had positive intraoperative microbial evidence of infection. Bacterial testing was mostly positive in fractures of the tibia (47.4\%) and the femur (27.5\%). Coagulase-negative Staphylococcus spp. were the most frequently detected (44.4\%) followed by mixed infections (18.7\%) and Staphylococcus aureus (10.5\%). Antibiograms revealed that $62.6 \%$ of our cases were cefuroxime sensitive; $87.7 \%$ were gentamicin sensitive. Only $10.5 \%$ showed resistance to both cefuroxime and gentamicin. There was no statistically significant difference of fusion rates between patients with different microbial species or different antibiograms.

Conclusion: Our data suggest that besides the high variety of different detected species, initial antibiotic treatment with a combination of systemic cefuroxime and local gentamicin-loaded bone cement is effective and in almost $90 \%$ the later determined microbial infection was sensitive to this treatment. Therefore, we recommend initial treatment according to this algorithm until specific antibiograms are available from intraoperatively acquired tissue samples.

Keywords: infected nonunions, antibiotic treatment, microbial spectrum, antibiogram

\section{Background}

Infected nonunions of the long bones remain a great challenge and are one of the most feared complications in the field of orthopedic and trauma surgery. ${ }^{1}$ Treatment frequently consists of repeated and radical surgical debridement in combination with prolonged antibiotic therapy. ${ }^{2}$ This results in a high risk of associated complications with recurrent infections, reduction of quality of life due to functional disabilities, and high socioeconomic costs. ${ }^{3-6}$ Not only patients with open fractures, but also patients with closed fractures and severe soft tissue damage are at risk for infection. ${ }^{7}$ The incidence 
of osteomyelitis after complex fractures is high, especially in open fractures with high grade tissue damage. ${ }^{8,9}$ Infection as one of the contributing etiological factors for the development of a nonunion has been underestimated in the past and hence microbiological testing should be performed more often. ${ }^{10}$ Staphylococcus aureus has been described as the main causative agent in up to $55 \%$ of osteomyelitis cases, ${ }^{11,12}$ but recently other species, such as Propionibacterium spp., have also been linked to implant-associated infections. ${ }^{13}$ Polymicrobial infections have been increasingly detected in peri-prosthetic infections and nonunions with poor clinical outcome and cause tremendous challenges for microbiological treatment. ${ }^{14,15}$ The development of an effective microbiological concept with initially started optimal calculated antibiotic therapy before availability of the specific bacterial species would result in a more reliable antimicrobial treatment strategy. This would decrease time and costs of treatment (eg, a reduction in hospital stay, follow-up treatment, and resources) and could improve patients' quality of life.

Therefore, the goal of this study was to evaluate both the bacterial spectrum and the antibiotic resistance as well as sensibility of patients suffering from a nonunion and a prior history of infection in order to optimize the initial antibiotic therapy starting with the first revision surgery and before availability of the results of microbiological intraoperative testing.

\section{Materials and methods}

\section{Patient recruitment}

All patients aged $\geq 18$ years undergoing revision surgery due to nonunion of long bones (humerus, radius, ulna, femur, tibia, fibula, clavicle) with a clinical history of infection between March 2010 and December 2015 were included in the current study. Approval was given by the ethical committee of the Ruprecht-Karls-University of Heidelberg, Germany (votum number S-262/2017). Exclusion criteria were corticosteroid medication (excluding hormonal contraception) or chemotherapy. All patients gave written informed consent to participate in the study. The collection of all patient-related data was performed retrospectively using a computerized databank. This included personal data of the patients (age and gender); information about the accident, such as fracture localization, classification, and soft tissue damage; medical history concerning infections and comorbidities according to Wawersik; ${ }^{16}$ treatment procedure; and laboratory tests. Preoperative condition of the patient was determined according to the classification of the American Society of Anesthesiologists (ASA). The ASA classification is accepted worldwide due to its straightforwardness - a higher
ASA score indicates higher morbidity and mortality of the patient. ${ }^{17}$

\section{Surgical procedure}

For infected nonunions, we performed a 2-step approach, according to the principles of the "diamond concept" 18 including the Masquelet technique. ${ }^{19}$ The first step of the procedure includes radical debridement of the infected site of the nonunion and application of a gentamicin-impregnated bone cement (Palacos ${ }^{\circledR} \mathrm{R}+\mathrm{G}$; $0.5 \mathrm{~g}$ gentamicin per $40 \mathrm{~g}$ cement) in the bone defect. Multiple bone and soft tissue samples were harvested for microbiological analysis. All patients received perioperative intravenous antibiotic treatment with cefuroxime, which was started immediately after microbiological samples were taken and continued for 4-6 weeks or until different antibiogram results were available. Systemic antibiotic dosing was carried out with the highest possible therapeutic doses (eg, $3 \times 1.5$ g cefuroxime per day) but individually adjusted to kidney function. If the antibiogram showed cefuroxime resistance, the antibiotic therapy was adjusted. After 6 weeks, the second step was performed. The gentamicin-impregnated bone cement (Palacos ${ }^{\circledR} \mathrm{R}+\mathrm{G}$; Heraeus Medical, Wehrheim, Germany) in the bone defect was removed and the nonunion was stabilized with a locking compression plate or intramedullary nailing with a gentamicin-coated implant (Expert Tibial Nail PROtect ${ }^{\circledR}$; Synthes GmbH, Oberdorf, Switzerland). The defect was filled with autologous bone graft taken from either the iliac crest or the contralateral femur using the Reamer-Irrigator-Aspirator ${ }^{\mathrm{TM}}$ (Synthes $\mathrm{GmbH}$ ). After this second step patients continued systemic antibiotic treatment for at least 2 weeks.

\section{Radiologic evaluation of bone fusion}

Bridging of the fracture callus was semi-quantitatively evaluated with a straightforward scoring system (0-2), modified and adapted from the radiographic score of Lane and Sandhu for fracture unions. ${ }^{20}$ At the latest available $\mathrm{X}$-rays and computed tomography scans the degree of bone fusion was rated according to the clearance of the fracture line $(2=$ complete bone fusion with absent fracture line, $1=$ incomplete bone fusion with partial fracture line, and $0=$ no bone fusion with full fracture line). Final analysis was performed in June 2017. Mean radiologic follow-up duration was 34.2 months $( \pm 19.9)$. Minimal follow-up duration (from revision surgery to latest available imaging) was 1 month.

\section{Microbiological procedures}

Tissue samples were processed according to the standardized setup of our microbiological department. Positive evidence for bacterial infection of the bone defect was defined if obligate 
pathogen bacteria were detected or $\geq 2$ independent tissue samples were positive for the same facultative pathogen bacteria (eg, Staphylococcus epidermidis or Corynebacterium spp., which are known as commensals of the skin). ${ }^{21}$ Tissue was further processed and cultivated in our microbiology department as previously described. ${ }^{22}$ Samples were spread onto porcelain mortar, followed by the addition of $1 \mathrm{~mL}$ of $0.9 \% \mathrm{NaCl}$. This suspension was inoculated onto Columbia 5\% sheep blood agar, chocolate agar, MacConkey agar, streptococcus-selective agar, Schaedler Neo Vanco $+5 \%$ sheep blood agar (all BioMérieux, Marcy-l'Étoile, France), and thioglycolate broth (BD Diagnostic Systems, San Jose, CA, USA), and then Gram staining was performed. Plates and broth were incubated until positive or for a maximum of 5 days at $36^{\circ} \mathrm{C}$ in $5 \% \mathrm{CO}_{2}$ or under anaerobic conditions. Identification of bacteria was done with a matrix-assisted laser desorption/ionization time-of-fight mass spectrometer (Microfex; Bruker Corporation, Billerica, MA, USA). Susceptibility testing was performed using a Vitek 2 microbial identification system (BioMérieux). ${ }^{22}$

\section{Statistical analysis}

Data entry was done with MS Excel, and statistical analysis was performed using SPSS for Windows 12.0 (SPSS Inc, Chicago, IL, USA). Complete data sets were available for 212 patients with nonunion and history of infection fulfilling the abovementioned selection criteria. Association between continuous and discrete variables was tested with Student's $t$-test. $P$-value was set at 0.05 .

\section{Results \\ Distribution of patient demographics and fracture types}

A total of 527 patients with a previously developed nonunion were surgically treated in our department between March 2010 and December 2015. Among them, 212 patients presented a history of infection and hence were selected for the present study. From all these patients, microbiological tissue samples were taken intraoperatively.

Microbiological testing of the tissues provided positive evidence for microbial infection in 171 patients $(80.7 \%)$. The majority of the patients were male $(72.5 \%, 124$ patients $)$. The mean age for the entire group was $52.1 \pm 13.9$ years; male patients had a slightly lower mean age $(50.8 \pm 13.5$ years $)$ compared to female patients $(55.3 \pm 14.3$ years $)$. There was no statistically significant difference between men and women.

Nonunion with positive bacterial evidence was mostly detected in fractures of the tibia ( $47.4 \% ; 81$ cases), followed by fractures of the femur $(27.5 \% ; 47$ cases) (Figure 1$)$. Classification of the initial fracture resulting in a nonunion revealed a majority of closed fractures $(51.5 \% ; 88$ cases), whereas open fractures were most often grade I (21.6\%; 37 cases). A detailed distribution of fracture types is presented in Figure 2. According to the ASA classification, most patients (46.2\%, 79 cases) were classified as ASA I, whereas $30.4 \%$ (52 cases) were ASA II, 19.9\% (34 cases) ASA III, and only $3.5 \%$ (6 cases) ASA IV.

\section{Microbial infection}

In case of a detected microbial infection, coagulase-negative Staphylococcus spp. were most frequently found (44.4\%; 76 cases) followed by mixed infections with $18.7 \%$ (32 cases) including combinations of $S$. aureus, S. epidermidis, Enterococcus faecalis, Escherichia coli, Acinetobacter lwoffii, or Raoultella ornithinolytica. Monoinfection with S. aureus was found in $10.5 \%$ (18 cases). Extremely rarely detected species were Citrobacter freundii, Candida parapsilosis, Pseudomonas stutzeri, or A. lwoffii. A detailed distribution of all different microbial species is shown in Table 1 . In 41 cases $(19.3 \%)$ of all our patients with history of infection

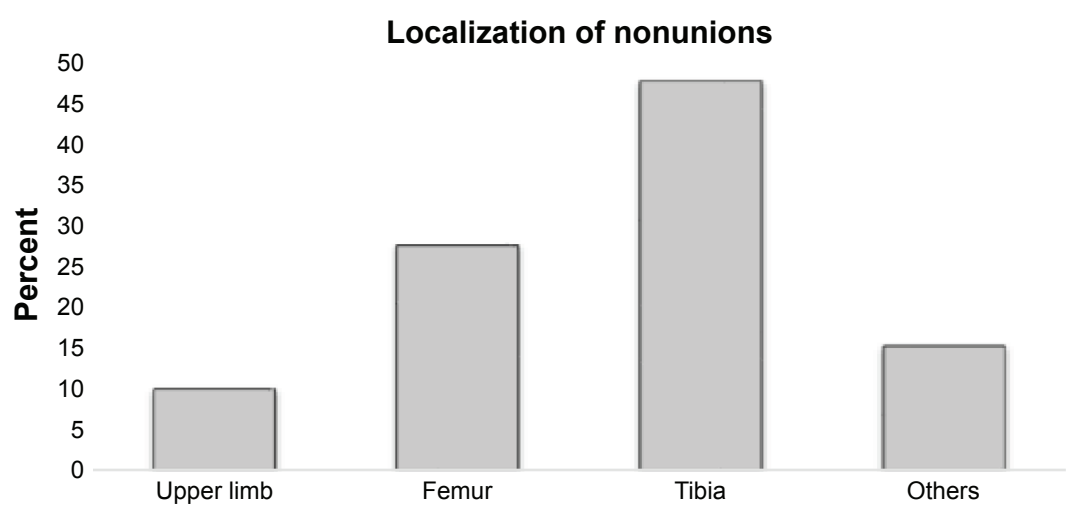

Figure I Distribution (in \%) of localizations of infected nonunions. 


\section{0}

Fracture types

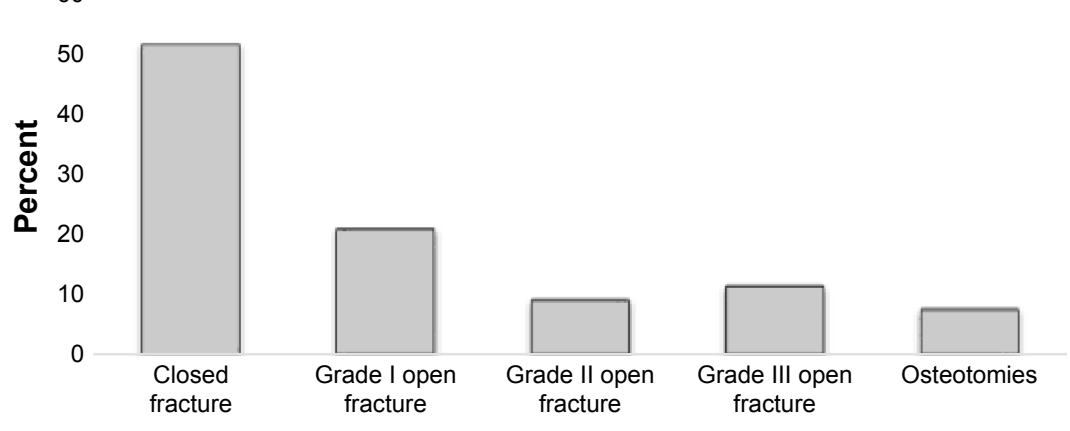

Figure 2 Distribution (in \%) of fracture types causing infected nonunions.

(212 patients), microbial presence could not be proven with microbiological testing.

Analysis of the antibiogram revealed that 107 (62.6\%) cases with detected microbial infection were cefuroxime sensitive. Of those, only 3 were gentamicin resistant. Fortysix cases (26.9\%) showed resistance against cefuroxime but were sensitive for gentamicin. Hence, a total of 150 cases (87.7\%) was gentamicin sensitive. Only 10.5\% (18 cases) of our cases with detected microbial infection showed resistance to both cefuroxime and gentamicin.

Cases which were cefuroxime sensitive (107; 62.6\%) showed complete bone fusion in $63.6 \%$ (68 cases), incomplete bone fusion in $17.7 \%$ (19 cases), and no bone fusion in $18.7 \%$ (20 cases) at final follow-up. In the gentamicin

Table I Detected species from tissue samples of all I7I infected nonunions with positive microbial evidence

\begin{tabular}{ll}
\hline Species & $\begin{array}{l}\text { Number of positive tissue } \\
\text { samples (N=I7I), n (\%) }\end{array}$ \\
\hline Coagulase-negative Staphylococcus spp. & $76(44.4)$ \\
Mixed infection & $32(18.7)$ \\
Staphylococcus aureus & $18(10.5)$ \\
Enterobacter cloacae & $10(5.8)$ \\
Bacillus spp. & $8(4.7)$ \\
Proprionibacterium spp. & $5(2.9)$ \\
Streptococcus spp. & $4(2.4)$ \\
Candida parapsilosis & $3(1.8)$ \\
Citrobacter freundii & $2(1.2)$ \\
Escherichia coli & $2(1.2)$ \\
Pseudomonas stutzeri & $2(1.2)$ \\
Acinetobacter Iwoffii & $\mathrm{I}(0.6)$ \\
Finegoldia magna & $\mathrm{I}(0.6)$ \\
Proteus mirabilis & $\mathrm{I}(0.6)$ \\
Clostridium tertium & $\mathrm{I}(0.6)$ \\
Peptoniphilus harei & $\mathrm{I}(0.6)$ \\
Clostridium clostridioforme & $\mathrm{I}(0.6)$ \\
Enterococcus faecalis & $\mathrm{I}(0.6)$ \\
Serratia marcescens & $\mathrm{I}(0.6)$ \\
Micrococcus luteus & $\mathrm{I}(0.6)$ \\
\hline
\end{tabular}

sensitive cases (150 patients; 87.7\%), 63.3\% (95 cases) achieved complete bone fusion, 16.7\% (25 cases) incomplete bone fusion, and 20.0\% (30 cases) no bone fusion. The 46 cases $(26.9 \%)$, which showed resistance against cefuroxime but were sensitive to gentamicin, showed $63 \%$ (29 cases) achieving complete bone fusion, 15.2\% (7 cases) incomplete bone fusion, and $21.7 \%$ (10 cases) no bone fusion at final follow-up. The 18 cases which were completely resistant to our antibiotic regime with cefuroxime and gentamicin, however, also achieved complete bone fusion in 11 cases $(61.1 \%)$ at final follow-up. Only 4 (22.2\%) of these patients demonstrated no bone fusion, and 3 patients $(16.7 \%)$ had incomplete bone fusion after 2 years. There was no statistically significant difference of the fusion rates between these 4 groups (cefuroxime-sensitive cases; gentamicinsensitive cases; cefuroxime- and gentamicin-sensitive cases; cefuroxime- and gentamicin-resistant cases).

For all nonunion patients with history of infection, $63.2 \%$ (108 cases) demonstrated full bone fusion. A representative case with clinical success and complete fusion is shown in Figure 3. Seventeen percent (29 cases) showed partial bone fusion, and 19.8\% (34 cases) showed no bone fusion as depicted in Figure 4. Analyzing the 41 patients where microbial presence could not be proven, 23 (56.1\%) showed complete bone fusion, $10(24.4 \%)$ incomplete bone fusion, and $8(19.5 \%)$ no bone fusion, without a statistically significant difference compared to the whole collective.

Regarding the 3 most frequently detected species, differences in fusion rates were not statistically significant (Figure 5).

\section{Discussion}

Fracture healing is a complex multistep process, which when disturbed may result in nonunion of the bone. ${ }^{22,23}$ Bacterial infection is one possible reason for impaired fracture 
A

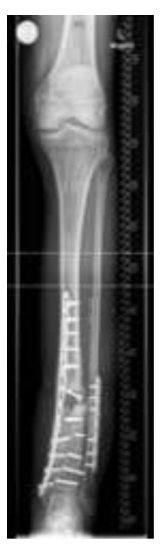

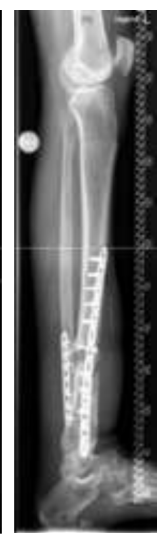

B

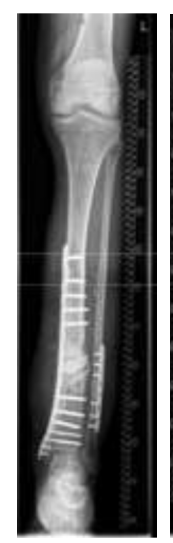

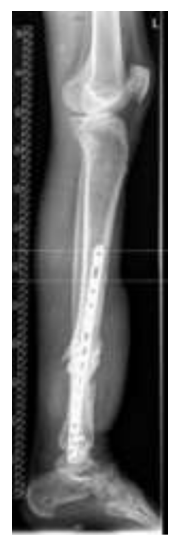

C

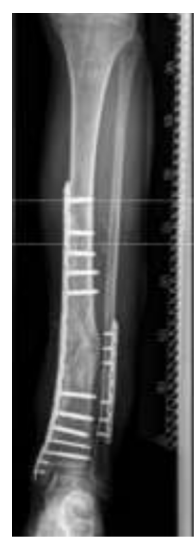

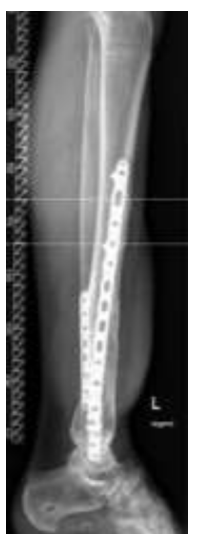

Figure 3 Successful nonunion treatment after Masquelet technique.

Notes: Nonunion of the distal tibia 18 months after fracture with microbiological evidence of coagulase-negative Staphylococcus spp. (A). Postoperative image after changing the osteosynthetic material together with extensive debridement and implantation of a bone cement spacer according to Masquelet technique step I (B). Final image after Masquelet step 2 with spacer removal, metaphyseal locking compression plate re-osteosynthesis and implantation of cancellous bone of the femur into the fracture gap. The patient showed complete fracture healing after 16 months (C).

healing and nonunion. ${ }^{22}$ Treatment of nonunions secondary to bacterial osteitis still represents a challenge in the field of orthopedic and trauma surgery. ${ }^{5,22}$ The aim of this study was to investigate the microbial spectrum as well as the antibiotic resistance and sensibility of all patients with nonunions and history of infection in order to optimize the initial antibiotic therapy.

Our results show that only $10.5 \%$ of all patients who were initially treated with the aforementioned antibiotic therapy suffered from a bacterial colonization that was not sensitive to this initially blind antibiotic regime. Though resistant to the later found bacterial species, this initial antibiotic treatment did not hamper the radiological outcome as there was no significant impact on bone fusion rates over time. Change of systemic antibiotic treatment as soon as the antibiogram was available may have contributed to this success. All patients showed good consolidation rates at final follow-up with $63 \%$ complete radiologic bone fusion and $17 \%$ at least partial bone fusion resulting in an overall radiologic success of $80 \%$ of the cases. Final success rates in the literature after revision surgery of nonunions are heterogeneous and difficult to compare but reach almost $90 \%$, depending on patient selection and surgical treatment strategies. ${ }^{24-26}$ Detailed clinical success rates, however, were not determined in the present study, since emphasis was on the microbiologic outcome.

Nonunions can be further divided according to their etiology. The present study only looked at infected nonunions, where bacterial infection is thought to be an important etiological cofactor causing insufficient bone growth. Percentages and types of microbial species in our large study
A

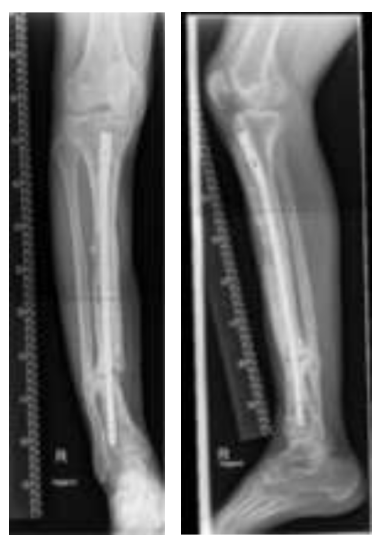

B

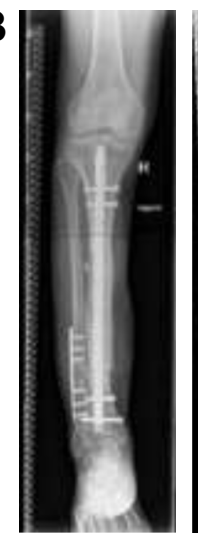

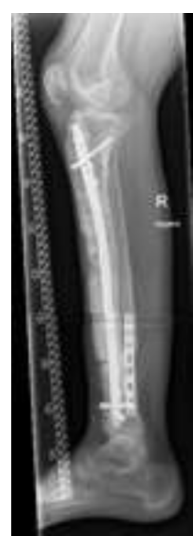
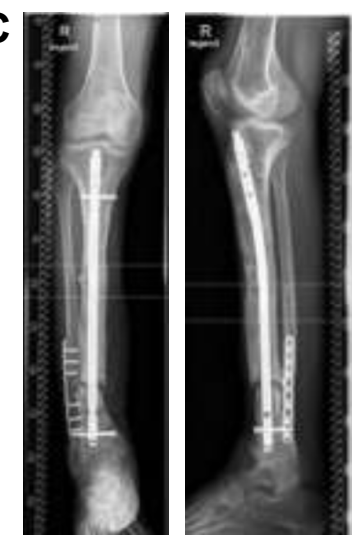
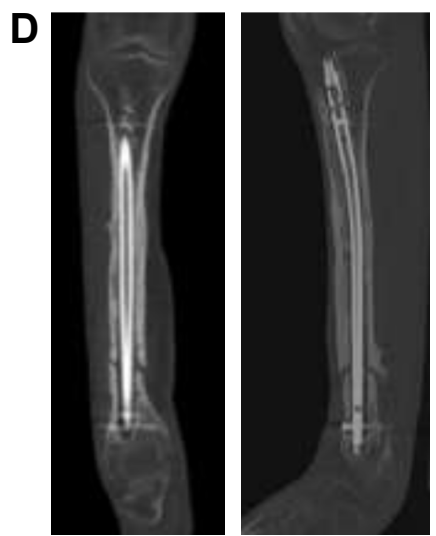

Figure 4 Unsuccessful nonunion treatment after Masquelet technique.

Notes: Nonunion of the distal tibia 20 months after fracture with microbiological evidence of a mixed infection (A). Postoperative image after changing the tibial nail and extensive debridement with implantation of a bone cement spacer according to Masquelet step I and additional implantation of a locking compression plate at the fibula (B). Masquelet step 2 was performed 6 weeks later with spacer removal and re-osteosynthesis using an Expert Tibial Nail PROtect ${ }^{\circledR}$ at the tibia together with cancellous bone. At 22 months after Masquelet step 2, the patient did not show fracture healing in X-rays (C) and computed tomography scan (D), yet. 


\section{Bone fusion rates}

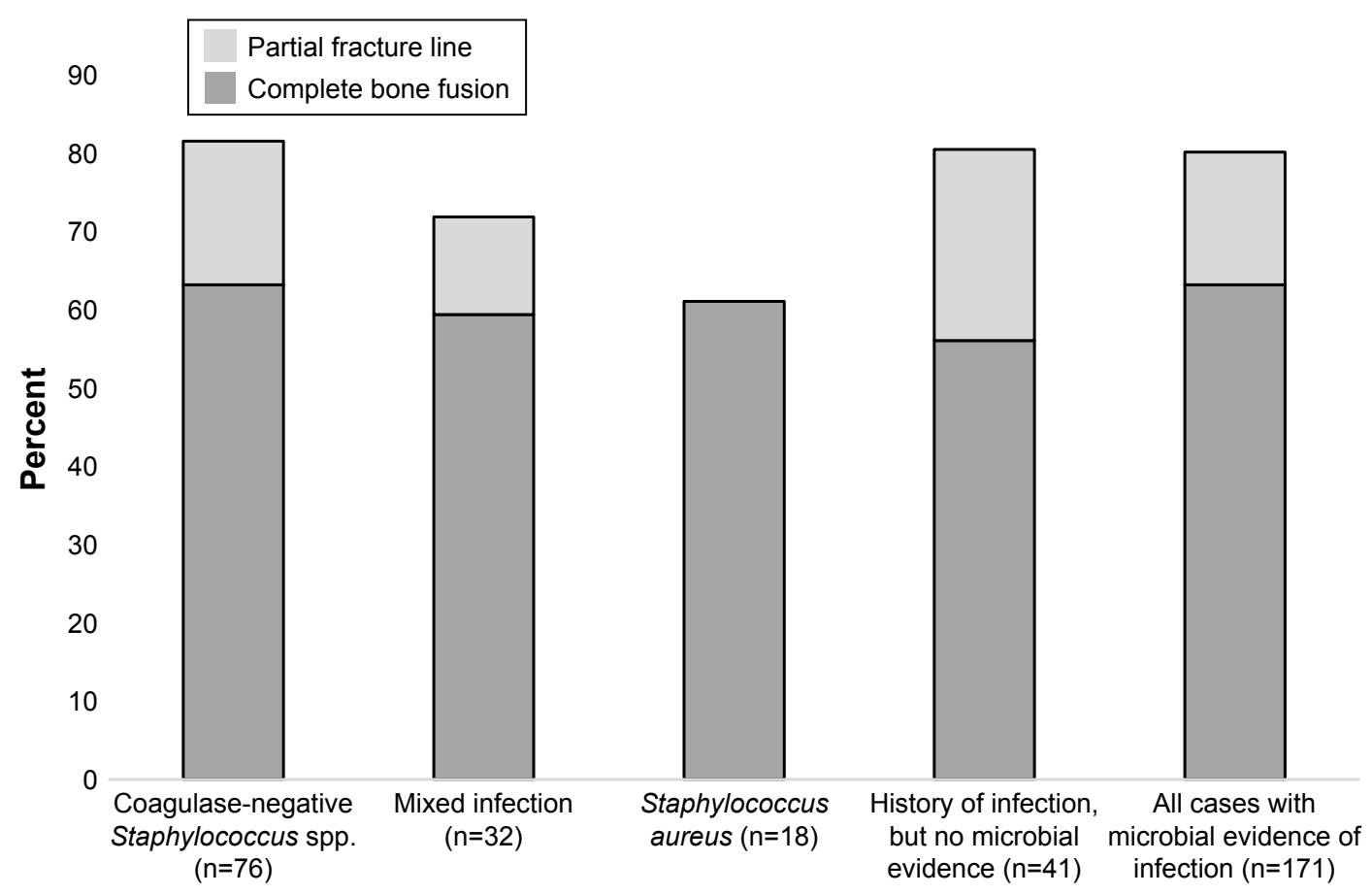

Figure 5 Fusion rates (in \%) of cases with infection with the 3 most frequently detected bacteria and cases without detected microbial infection compared to all I7I cases of infected nonunion with positive microbial evidence.

Note: Lower part of each row shows complete bone fusion rate with absent fracture line, upper part depicts rate of incomplete bone fusion with partial fracture line.

group mainly confirm other reports in the literature where coagulase-negative staphylococci and S. aureus were detected most frequently. ${ }^{10,27}$ The reason why monoinfection with $S$. aureus was slightly less frequent in our series cannot be completely answered but might be related to a higher percentage of patients with multiple foregoing surgeries in other hospitals and more challenging soft tissue problems resulting in a higher percentage of mixed infections and infections with facultative pathogen bacteria of the skin.

Nonunion is a complex disease with multiple etiologies and it is difficult to define the critical problem of each case. ${ }^{10}$ Other important factors are mechanical instability due to insufficient osteosynthesis, local avital bone, and individual host factors such as smoking ${ }^{28}$ or other abnormalities. ${ }^{29}$ Mechanical insufficiency and dead bone have been determined as the most important etiological factors. ${ }^{10}$ To address this, radical debridement as the most important therapy against osteomyelitis ${ }^{30}$ is the basic principle of our treatment algorithm. To what extent further infection influenced the outcome cannot be fully answered by the present study, as we only analyzed cases with a history of infection. The high percentage of cases with a history of infection $(40.2 \%$; 212 of 527 cases), which was found if looked at all nonunion patients treated in our department in the mentioned 5-year study time frame, suggests that infection is an important cofactor. The main etiology of the nonunions of our collective, however, cannot be identified by our study design and this was also not the goal of our study. Whether infected nonunions had different fusion rates than noninfected nonunions cannot be answered, as we only analyzed cases with a history of infection. It is most likely that at least a considerable part of the 41 cases, without positive microbial tissue samples, still had an infected nonunion which was just not detected at the first revision surgery. In order to give these patients - based on their history of high likelihood of persistent infection - the best possible treatment, our algorithm continued postoperative systemic antibiotic treatment with cefuroxime in addition to the gentamicin-loaded bone cement. Our antibiogram data of the other $80 \%$ of the cases, where the bacteria could be detected, support this strategy as almost $90 \%$ were sensitive to either cefuroxime or gentamicin. Hence, the presented strategy with gentamicin and cefuroxime would have improved infection therapy in most of the cases where infection was still active but could not be proven by microbiology.

The result that both antibiotic resistance and the species of bacteria did not significantly influence the final radiologic outcome with similar fusion rates can be interpreted 
differently. One explanation could be that infection was less important than the other abovementioned etiologies, which were all addressed by our surgical algorithm using the diamond concept and Masquelet technique. ${ }^{18,19}$ Another explanation, however, is that our antibiotic regime was effective or at least sufficient for all treated patients and this is supported by several points as follows. The combination of systemic cefuroxime and local gentamicin already provided initial sensitive antibiotic treatment for almost $90 \%$ of all treated patients. Additionally, local application of high-dose gentamicin results in the highest possible local antibiotic concentrations $^{31-34}$ without systemic intoxication, ${ }^{35}$ which may result in sufficient local antimicrobial effects even if gentamicin resistance was evident in the antibiogram due to the fact that the local concentrations widely exceed the minimal inhibitory concentrations or minimal bactericidal concentrations of the bacteria. ${ }^{36-39}$ Another concept involves biodegradable local drug delivery systems combined with fracture fixation devices such as intramedullary nails coated with poly-(D,L-lactide) and gentamicin. This coating significantly reduced bacterial adhesion on the implant surface $e^{40-42}$ and resulted in the first convincing clinical results. ${ }^{5,42,43}$ Consequently, our patients with infected tibial nonunions also received gentamicin-coated intramedullary nails.

Biofilm development is an important problem known for continuous infection together with osteosynthetic or prosthetic

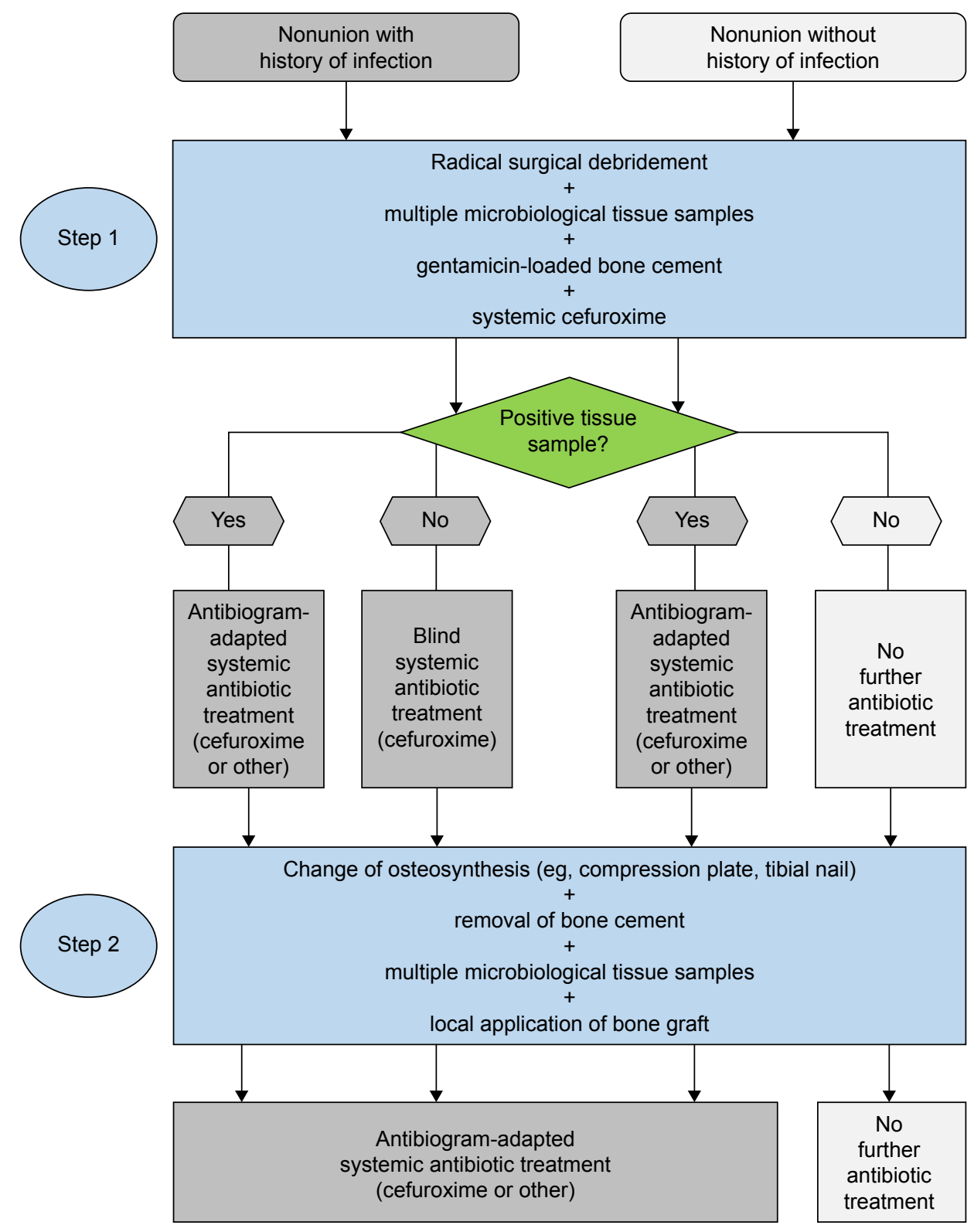

Figure $\mathbf{6}$ Schematic algorithm of Masquelet step I and 2 with peri- and postoperative antibiotic treatment. 
implants $^{44}$ and significantly reduces the efficacy of antibiotics. ${ }^{45}$ Bacteria persist in this biofilm colony as they stick to each other and adhere to the implant protected by the production of extracellular polymeric matrix. ${ }^{46,47}$ Local antibiotics such as gentamicin-loaded bone cement may attack biofilms more effectively than systemic antibiotics due to higher locally available doses, ${ }^{48,49}$ but nonetheless radical surgical debridement and changing the implant are required and are part of the administered diamond concept. We also recommend partially covering the metal implant with additional bone cement if possible to further reduce biofilms on the implant.

Another important aspect is that antibiotics were adjusted according to the antibiogram as soon as the antibiogram was available. So, for the presented $10.5 \%$ cases with resistance against cefuroxime and gentamicin, systemic antibiotic treatment was adjusted later on at a mean of 5 days after the first surgery. The cefuroxime-resistant cases (37.4\%) were then changed into antibiogram-adapted systemic antibiotics which were continued for up to 6 weeks. As the majority (62.6\%) of all our analyzed cases was cefuroxime sensitive, we recommend blind initial postoperative systemic treatment with cefuroxime.

Taken together, we believe that the used algorithm (Figure 6) utilizing an initial antibiotic treatment including both systemic cefuroxime and local gentamicin is effective and should be the standard for nonunions with a history of infection. Since infection cannot be ruled out in cases without a history of infection, we recommend taking multiple microbiological tissue samples during surgical treatment of all nonunions as well as the use of gentamicin-loaded bone cement together with systemic cefuroxime until microbiology results are available. If the microbiology results are negative, systemic cefuroxime should be discontinued.

The strength of the present study is the large number of patients and full documentation of clinical and microbiological data. To our knowledge, this is the first study evaluating the full spectrum of microbial species in such a large number of patients with nonunions in the context of radiological outcome. Limitation of the study was lack of a comparison group without history of infection.

\section{Conclusion}

Our data suggest that initial antibiotic treatment with systemic cefuroxime and local gentamicin-loaded bone cement before availability of microbiological results is effective, and in almost $90 \%$ the detected microbial infection is already sensitive to this regime. Therefore, we recommend initial treatment according to this algorithm at least in cases of nonunions suspicious for or with a history of infection. The high percentage of proven bacterial infection in case of a nonunion found in our patients should be carefully considered and hence microbiological testing should be routinely performed in all nonunion revision surgeries.

\section{Acknowledgment}

This study was internally funded by the Department of Orthopaedics, Trauma Surgery and Paraplegiology, Heidelberg University Hospital.

\section{Disclosure}

Gerhard Schmidmaier has a patent registration for the gentamicin coating of the Expert Tibial Nail PROtect ${ }^{\circledR}$. The other authors report no conflicts of interest in this work.

\section{References}

1. Struijs PA, Poolman RW, Bhandari M. Infected nonunion of the long bones. J Orthop Trauma. 2007;21(7):507-511.

2. Moghaddam A, Zimmermann G, Hammer K, Bruckner T, Grutzner PA, von Recum J. Cigarette smoking influences the clinical and occupational outcome of patients with tibial shaft fractures. Injury. 2011; 42(12): 1435-1442.

3. Poultsides LA, Liaropoulos LL, Malizos KN. The socioeconomic impact of musculoskeletal infections. J Bone Joint Surg Am. 2010; 92(11):e13.

4. Stengel D, Bauwens K, Sehouli J, Ekkernkamp A, Porzsolt F. Systematic review and meta-analysis of antibiotic therapy for bone and joint infections. Lancet Infect Dis. 2001;1(3):175-188.

5. Schmidmaier G, Lucke M, Wildemann B, Haas NP, Raschke M. Prophylaxis and treatment of implant-related infections by antibioticcoated implants: a review. Injury. 2006;37(Suppl 2):S105-S112.

6. Moghaddam A, Elleser C, Biglari B, Wentzensen A, Zimmermann G. Clinical application of BMP 7 in long bone non-unions. Arch Orthop Trauma Surg. 2010;130(1):71-76.

7. Gustilo RB, Anderson JT. JSBS classics. Prevention of infection in the treatment of one thousand and twenty-five open fractures of long bones. Retrospective and prospective analyses. J Bone Joint Surg Am. 2002;84A(4):682.

8. Heppert V, Wentzensen A. [Treatment of soft tissue damage-definitive management]. Langenbecks Arch Chir Suppl Kongressbd. 1998;115: 964-967. German.

9. Schmidt HG, Wittek F, Fink B, Buck-Gramcko U. [Treatment of chronic osteitis of the tibia]. Unfallchirurg. 1992;95(11):566-573. German.

10. Mills L, Tsang J, Hopper G, Keenan G, Simpson AH. The multifactorial aetiology of fracture nonunion and the importance of searching for latent infection. Bone Joint Res. 2016;5(10):512-519.

11. Lindsey BA, Clovis NB, Smith ES, Salihu S, Hubbard DF. An animal model for open femur fracture and osteomyelitis: part I. J Orthop Res. 2010;28(1):38-42.

12. Arciola CR, Campoccia D, Speziale P, Montanaro L, Costerton JW. Biofilm formation in Staphylococcus implant infections. A review of molecular mechanisms and implications for biofilm-resistant materials. Biomaterials. 2012;33(26):5967-5982.

13. Aubin GG, Portillo ME, Trampuz A, Corvec S. Propionibacterium acnes, an emerging pathogen: from acne to implant-infections, from phylotype to resistance. Med Mal Infect. 2014;44(6):241-250.

14. Tan TL, Kheir MM, Tan DD, Parvizi J. Polymicrobial periprosthetic joint infections: outcome of treatment and identification of risk factors. J Bone Joint Surg Am. 2016;98(24):2082-2088.

15. Chadayammuri V, Herbert B, Hao J, et al. Factors associated with adverse postoperative outcomes in patients with long bone post-traumatic osteomyelitis. Eur J Orthop Surg Traumatol. Epub 2017 Apr 28. 
16. Wawersik J. [Data processing in anesthesia based on the example of documentation-justified anesthesia protocol]. Z Prakt Anasth. 1970; 5(1):6-28. German.

17. Keats AS. The ASA classification of physical status - a recapitulation. Anesthesiology. 1978;49(4):233-236.

18. Giannoudis PV, Einhorn TA, Schmidmaier G, Marsh D. The diamond concept - open questions. Injury. 2008;39(Suppl 2):S5-S8.

19. Masquelet AC, Obert L. [Induced membrane technique for bone defects in the hand and wrist]. Chir Main. 2010;29(Suppl 1):S221-S224. French.

20. Lane JM, Sandhu HS. Current approaches to experimental bone grafting. Orthop Clin North Am. 1987;18(2):213-225.

21. Grice EA, Segre JA. The skin microbiome. Nat Rev Microbiol. 2011; 9(4):244-253.

22. Dapunt U, Spranger O, Gantz S, et al. Are atrophic long-bone nonunions associated with low-grade infections? Ther Clin Risk Manag. 2015;11:1843-1852.

23. Ai-Aql ZS, Alagl AS, Graves DT, Gerstenfeld LC, Einhorn TA. Molecular mechanisms controlling bone formation during fracture healing and distraction osteogenesis. J Dent Res. 2008;87(2):107-118.

24. Giannoudis PV, Harwood PJ, Tosounidis T, Kanakaris NK. Restoration of long bone defects treated with the induced membrane technique: protocol and outcomes. Injury. 2016;47(Suppl 6):S53-S61.

25. Muhlhausser J, Winkler J, Babst R, Beeres FJP. Infected tibia defect fractures treated with the Masquelet technique. Medicine (Baltimore). 2017;96(20):e6948.

26. Morelli I, Drago L, George DA, Gallazzi E, Scarponi S, Romano CL. Masquelet technique: myth or reality? A systematic review and metaanalysis. Injury. 2016;47(Suppl 6):S68-S76.

27. Lew DP, Waldvogel FA. Osteomyelitis. Lancet. 2004;364(9431): 369-379.

28. Bender D, Haubruck P, Boxriker S, Korff S, Schmidmaier G, Moghaddam A. Validity of subjective smoking status in orthopedic patients. Ther Clin Risk Manag. 2015;11:1297-1303.

29. Serbest S, Tiftikci U, Tosun HB, Gumustas SA, Uludag A. Is there a relationship between fracture healing and mean platelet volume? Ther Clin Risk Manag. 2016;12:1095-1099.

30. Walter G, Kemmerer M, Kappler C, Hoffmann R. Treatment algorithms for chronic osteomyelitis. Dtsch Arztebl Int. 2012;109(14):257-264.

31. Regis D, Sandri A, Samaila E, Benini A, Bondi M, Magnan B. Release of gentamicin and vancomycin from preformed spacers in infected total hip arthroplasties: measurement of concentrations and inhibitory activity in patients' drainage fluids and serum. Sci World J. 2013;2013:752184.

32. Chang Y, Tai CL, Hsieh PH, Ueng SW. Gentamicin in bone cement: a potentially more effective prophylactic measure of infection in joint arthroplasty. Bone Joint Res. 2013;2(10):220-226.

33. Anagnostakos K, Meyer C. Antibiotic elution from hip and knee acrylic bone cement spacers: a systematic review. Biomed Res Int. 2017; 2017:4657874.
34. Zalavras CG, Patzakis MJ, Holtom P. Local antibiotic therapy in the treatment of open fractures and osteomyelitis. Clin Orthop Relat Res. 2004;427:86-93.

35. Moghaddam A, Graeser V, Westhauser F, et al. Patients' safety: is there a systemic release of gentamicin by gentamicin-coated tibia nails in clinical use? Ther Clin Risk Manag. 2016;12:1387-1393.

36. Webb JC, Spencer RF. The role of polymethylmethacrylate bone cement in modern orthopaedic surgery. J Bone Joint Surg Br. 2007; 89(7):851-857.

37. Balint L, Koos Z, Horvath G, Szabo G. Detection of gentamicin emission from bone cement in the early postoperative period following total hip arthroplasty. Orthopedics. 2006;29(5):432-436.

38. Bunetel L, Segui A, Cormier M, Percheron E, Langlais F. Release of gentamicin from acrylic bone cement. Clin Pharmacokinet. 1989;17(4): 291-297.

39. Kanellakopoulou K, Giamarellos-Bourboulis EJ. Carrier systems for the local delivery of antibiotics in bone infections. Drugs. 2000;59(6): 1223-1232.

40. Vester H, Wildemann B, Schmidmaier G, Stockle U, Lucke M. Gentamycin delivered from a PDLLA coating of metallic implants: in vivo and in vitro characterisation for local prophylaxis of implant-related osteomyelitis. Injury. 2010;41(10):1053-1059.

41. Lucke M, Wildemann B, Sadoni S, et al. Systemic versus local application of gentamicin in prophylaxis of implant-related osteomyelitis in a rat model. Bone. 2005;36(5):770-778.

42. Schmidmaier G, Kerstan M, Schwabe P, Sudkamp N, Raschke M. Clinical experiences in the use of a gentamicin-coated titanium nail in tibia fractures. Injury. 2017;48(10):2235-2241.

43. Fuchs T, Stange R, Schmidmaier G, Raschke MJ. The use of gentamicincoated nails in the tibia: preliminary results of a prospective study. Arch Orthop Trauma Surg. 2011;131(10):1419-1425.

44. Getzlaf MA, Lewallen EA, Kremers HM, et al. Multi-disciplinary antimicrobial strategies for improving orthopaedic implants to prevent prosthetic joint infections in hip and knee. J Orthop Res. 2016; 34(2):177-186.

45. Costerton JW. Biofilm theory can guide the treatment of device-related orthopaedic infections. Clin Orthop Relat Res. 2005;437:7-11.

46. Marais LC, Ferreira N, Aldous C, Le Roux TL. The outcome of treatment of chronic osteomyelitis according to an integrated approach Strategies Trauma Limb Reconstr. 2016;11(2):135-142.

47. Shen Y, Zhu W, Chen C, Nie Y, Lin X. Biofilm formation in attached microalgal reactors. Bioprocess Biosyst Eng. 2016;39(8):1281-1288.

48. Zahar A, Hannah P. [Addition of antibiotics to bone cement for septic prosthesis exchange]. Oper Orthop Traumatol. 2016;28(2):138-144. German.

49. Kuhn KD, Renz N, Trampuz A. [Local antibiotic therapy]. Unfallchirurg. 2017;120(7):561-572. German.
Therapeutics and Clinical Risk Management

\section{Publish your work in this journal}

Therapeutics and Clinical Risk Management is an international, peerreviewed journal of clinical therapeutics and risk management, focusing on concise rapid reporting of clinical studies in all therapeutic areas, outcomes, safety, and programs for the effective, safe, and sustained use of medicines. This journal is indexed on PubMed Central, CAS,

\section{Dovepress}

EMBase, Scopus and the Elsevier Bibliographic databases. The manuscript management system is completely online and includes a very quick and fair peer-review system, which is all easy to use. Visit http://www.dovepress.com/testimonials.php to read real quotes from published authors. 